\title{
Fachbeitrag
}

Wolfgang Giella

\section{Die neue ZHAW Hochschulbibliothek in Winterthur: Eine aktivitätsbasierte Raumkonzeption der Lernlandschaft}

DOI 10.1515/abitech-2015-0036

Zusammenfassung: In atemberaubend kurzer Zeit entstand mit der neuen Hochschulbibliothek der Zürcher Hochschule für Angewandte Wissenschaften (ZHAW) in Winterthur eine der modernsten Fachhochschulbibliotheken Europas. Der neue Bibliotheksstandort befindet sich in einem denkmalgeschützten Industriegebäude und weist rund 4620 Quadratmeter Bruttonutzfläche auf. Zusätzlich entstand eine Lernlandschaft von über 1700 Quadratmetern. Ihre aktivitätsorientierte Konzipierung sowie ihr Betrieb und erste Erfahrungen bilden einen Schwerpunkt dieses Beitrags.

Schlüsselwörter: Aktivitätsbasiertes Planen, Bibliotheksbau, Lernformen

Abstract: This article describes the building project of the new university library at the Zurich University for Applied Sciences (ZHAW). In an extremely short amount of time one of the most state-of-the-art university libraries in Europe has come to life. The new library which measures approximately 4620 square metres was built inside a historical, listed industrial facility. It includes a learning landscape of more than 1700 square metres. Its activitybased conception and operation, as well as first impressions, are the core theme of this article.

Keywords: Activity-Based Planning, Learning Area, Library Building Construction

\section{Kurze Entstehungsgeschichte der Schweizer Fachhochschulen sowie der Zürcher Hochschule für Angewandte Wissenschaften (ZHAW)}

In den 1990er Jahren wurden die Weichen für den Aufbau der Schweizer Fachhochschulen gestellt. Grundlage war das Fachhochschulgesetz vom 6. Oktober 1995. ${ }^{1}$ Diesem folgten die kantonalen Anschlussgesetze sowie die interkantonalen Vereinbarungen. Die Fachhochschulen befanden sich von Anfang an unter Bundeshoheit, weil - als Teil der erweiterten Berufsbildung - die Berufsbildung weitgehend vom Bund geregelt wird. Wie schwierig sich der Prozess gestaltete, zeigt folgende Pressemitteilung vom April 2003:

„Die Integration der Fachhochschulen ins schweizerische Hochschulnetz kommt voran. (...) Der Bundesrat hatte den neugegründeten Fachhochschulen im Jahre 1998 eine befristete Genehmigung bis Ende 2003 ausgesprochen. Die inhaltlichen Rahmenvorgaben des Bundes informieren über die materiellen Voraussetzungen für die Erneuerung der Genehmigung sowie über den Ablauf des Genehmigungsprozesses. Sie wurden durch das Bundesamt für Berufsbildung und Technologie (BBT) in enger Zusammenarbeit mit den Trägern der Fachhochschulen erarbeitet und sollen die Grundlage für eine unbefristete Genehmigung (...) bilden.“2

Nach Verabschiedung des FH-Gesetzes dauerte es noch volle drei Jahre, bis alle Voraussetzungen geschaffen waren, dass der Bundesrat erst einmal befristete Genehmigungen aussprechen konnte.

Im Jahr 2003 erfolgten im Kanton Zürich Strukturbereinigungen innerhalb der Zürcher Fachhochschule. Es wurden aus den bestehenden Institutionen drei rechtlich selbstständige staatliche Hochschulen innerhalb der ZFH gebildet, die Zürcher Hochschule der Künste (ZHdK), die Pädagogische Hochschule Zürich (PHZH) sowie die ZHAW.

\footnotetext{
1 Online einsehbar unter: http://www.admin.ch/opc/de/classifiedcompilation/19950279/index.html (06.09.2015).

2 Fachhochschulen auf der Zielgeraden. Pressemitteilung des BBT. RessortleitungThomasBaumeler, Kommunikation HelenStotzer.Link: https://www.admin.ch/cp/d/3e955cc8_1@presse1.admin.ch.html (19.08.2015).
} 
Tabelle 1: Organisatorische Zuordnungen der Hochschulfusion im Kanton Zürich

\begin{tabular}{|c|c|c|c|}
\hline $\begin{array}{l}\text { Vorgängerorganisationen } \\
\text { (Hochschule = HS) }\end{array}$ & ZHdK & PHZH & ZHAW \\
\hline HS für Gestaltung und Kunst (HGKZ) & $x$ & & \\
\hline HS für Musik und Theater (HMT) & $x$ & & \\
\hline HS Soziale Arbeit Zürich (HSSAZ) & & & $\mathrm{X}$ \\
\hline HS für Angewandte Psychologie (IAP) & & & $x$ \\
\hline HS Wädenswil (HSW) & & & $\mathrm{X}$ \\
\hline Pädagogische HS Zürich & & $x$ & \\
\hline HS Zürich ${ }^{3}$ & & & \\
\hline HS für Heilpädagogik ${ }^{4}$ & & & \\
\hline Technikum Winterthur Ingenieursschule (TWI) & & & $x$ \\
\hline Dolmetscherschule Zürich (DOZ) & & & $\mathrm{X}$ \\
\hline $\begin{array}{l}\text { Höhere Wirtschafts- und Verwaltungsschule } \\
\text { Zürich (HWV) }\end{array}$ & & & $\mathrm{x}$ \\
\hline
\end{tabular}

Im Jahr 2007 setzte der Fachhochschulrat des Kantons Zürich die Hochschulleitung der ZHAW ein.

Heute besteht die ZHAW aus acht getrennten Departementen, verteilt auf drei Städte des Kantons Zürich. Der Jahresbericht 2014 zeigt folgendes Bild:

Tabelle 2: Die Departemente der ZHAW nach Standorten heute, in Klammern jeweils die Anzahl der Studierenden

\begin{tabular}{ll}
\hline Winterthur & $\begin{array}{l}\text { Architektur, Gestaltung und Bauingenieurswesen (525) } \\
\text { Gesundheit (1354) } \\
\text { Angewandte Linguistik (769) } \\
\text { School of Engineering (1981) } \\
\text { School of Management and Law (3888) }\end{array}$ \\
\hline Zürich & Soziale Arbeit (762) \\
& Angewandte Psychologie (418) \\
\hline Wädenswil & Life Science and Facility Management (1489) \\
\hline
\end{tabular}

Insgesamt beschäftigte die ZHAW 20142984 Mitarbeitende und bot 26 Bachelor- und 14 Masterstudiengänge, in der Weiterbildung 47 MAS/EMBA, 155 CAS und 20 DAS an.

3 Die HS Zürich ist heute eine eigene kleine Wirtschafts-FH auf dem Platz Zürich, gehört aber zur ZFH.

4 Die Heilpädagogische HS ist ebenfalls ein eigenständiges $\mathrm{ZFH}$ Mitglied.

5 Wie alle Lerninstitutionen schmückte sich auch das TWI mit berühmten Lehrern. Der berühmteste unter ihnen ist sicher Albert Einstein, der vom 16. Mai bis im Herbst 1901 als Aushilfslehrer seine erste Stellung überhaupt ausgerechnet am Technikum Winterthur antrat. Von hier aus erlangte er seine Festanstellung am Patentamt in Bern, wo er 1902 die Arbeit aufnahm.

6 Studierendenzahlen (Bachelor- und Masterstudierende) sowie die Angaben über das geleistete Studienangebot stammen aus dem Jahresbericht 2014 der ZHAW: http://zhaw.ch/de/ueber-uns/zahlen-undfakten/jahresbericht (16.08.2015).

\section{Die ZHAW Hochschulbibliothek (HSB)}

Die HSB betrieb bis Ende 2014 an jedem Departement eine Bibliothek. Die Teams - von der klassischen One-PersonLibrary bis zu mittelgroßen Teams - gewährleisteten Studierenden, Dozierenden, Besuchern und Forschenden sämtliche Bibliotheksdienstleistungen inklusive eines differenzierten Schulungsangebots. Der Arbeitsalltag an den Standorten war sehr breit und nötigte allen Mitarbeitenden eine hohe fachliche Kompetenz ab. Zentral wurden die klassifikatorische Erschließung (DDC), der Erwerb von E-Medien sowie sämtliche Leitungs- und Stabsarbeiten geleistet.

Die Neubauprojekte der HSB in Winterthur als auch der neue Campus in Zürich veränderten binnen kurzer Zeit die Betriebsstruktur der Bibliothek stark. Die bisherigen fünf Standorte in Winterthur und die beiden Zürcher Bibliotheken, also insgesamt sieben Standorte, wurden $\mathrm{zu}$ zwei Standorten zusammengelegt. In Winterthur arbeiten die Mitarbeitenden neu in einem Kernteam mit, das eine höhere Spezialisierung von ihnen verlangt. Gleichzeitig bleibt die Arbeit vielfältig, da sie nach wie vor noch in mindestens einem anderen Team mitarbeiten.

\section{Das Bauprojekt}

Die ZHAW wurde schon früh von ihrem Erfolg überrollt, denn sie wuchs sehr stark und wächst weiterhin. Die strategische Entwicklung einer Hochschule, die aus einer Fusion mit Nachwehen entstanden ist und deren Einheiten weiterhin unterschiedlich stark wachsen, ist in allen Belangen und für alle Beteiligten eine riesige Herausforderung. Das klassische Merkmal einer solchen Phase ist die Unbeständigkeit. Neben den üblichen strategischen und operativen Arbeiten, die Universitäten und Hochschulen generell umtreiben, generiert das starke Wachstum für die Evaluierung mittel- und langfristiger Bedarfe gefährliche Bruchlinien zwischen Strategiearbeiten und operativer Realität. Eine solche Bruchlinie bildet die Raumplanung.

Am 12. Mai 2010 legte das Facility Management (FM) der ZHAW auf über 140 Seiten eine interne Raumentwicklungsstudie vor. Ein Teil dieser Studie zeichnete auch eine räumliche Bedarfsentwicklung der HSB als zentrale Einheit in Winterthur auf. Obschon die Wachstumszahlen von 2008 die Grundlage der Studie bildeten, mussten alle Angaben bibliotheksseitig neu errechnet werden. Auch hier hatte die Realität schon zwei Jahre später die kühnsten Wachstumsprognosen Lügen gestraft. Erschwerend hinzu kam, dass sich das FM noch sehr nahe am Typus 
der klassischen „Schülerbibliothek“ orientierte, was eine der Folgen aus der oben beschriebenen relativ jungen Entwicklungsgeschichte der schweizerischen Fachhochschulen war. Die Bedarfe, die Anspruchsgruppen und die wissenschaftlichen Erfordernisse sind jedoch auch an einer Fachhochschulbibliothek nicht sonderlich anders als an Universitätsbibliotheken.

\section{Areal- und Gebåudegeschichte}

Fährt man mit der Bahn von Zürich kommend in Winterthur ein, liegt auf der linken Seite das sogenannte Sulzerareal, ein großes Industrieareal. Eines der prägnantesten Gebäude, das durch Länge, Größe und rote Eternitplatten sowie mächtige Fensterfronten gleich ins Auge springt, ist das Gebäude 87, auch als Halle 87 oder in der Stadt Winterthur als Event-Halle bekannt.

Die von der Bahn aus gut sichtbare rund 25 Meter hohe und 100 Meter gerade Fassade des Gebäudes birgt den zum Industrieareal hin konvexen Rundbau mit seinen rund vierzig Metern Tiefe. Die Fassadenstruktur erinnert auch den Laien stark an Industriebauten der Bauhaus-Ära. Für Winterthur und den Kanton Zürich steht dieses Gebäude in der Tat als wichtigster Zeuge des Neuen Bauens der Schweiz. ${ }^{7}$

Dieses Industrieareal hat eine 170-jährige Geschichte und ist somit überregional von wirtschaftshistorischer Bedeutung. Im Jahr 1834 errichteten die Gebrüder Salomon und Johann Jakob Sulzer hier eine Eisengießerei, welche zuerst Feuerspritzen, Pressen sowie Pumpen produzierte. Später folgten Maschinen für die Textilindustrie, dann bald Heizungen und Dampfkessel. Um 1854 beschäftige die Firma Sulzer schon 500 Personen.

Unter Charles Brown, dem neurekrutierten technischen Betriebsleiter aus England, baute sich Sulzer ein neues Standbein auf. Auf der Weltausstellung in Paris 1867 wurde die Volldampfmaschine der Firma prämiert. Das war der Eintritt Sulzers in den Weltmarkt, was wiederum zur Folge hatte, dass vermehrt auch ausländische Studenten für ihr Firmenpraktikum nach Winterthur kamen. Einer dieser Studenten, der 1879 sein Praktikum in Winterthur absolvierte, verkaufte Sulzer wenige Jahre später eine Lizenz seiner bahnbrechenden Erfindung, die heute noch seinem Namen trägt: Es war Rudolf Diesel. 1898 nahm Sulzer den ersten Dieselmotor in Betrieb und

7 Einen guten Kurzüberblick zum Neuen Bauen in der Schweiz hat Dorothee Huber im Historischen Lexikon der Schweiz verfasst; online einsehbar unter: http://www.hls-dhs-dss.ch/textes/d/D11188. php (13.09.2015).

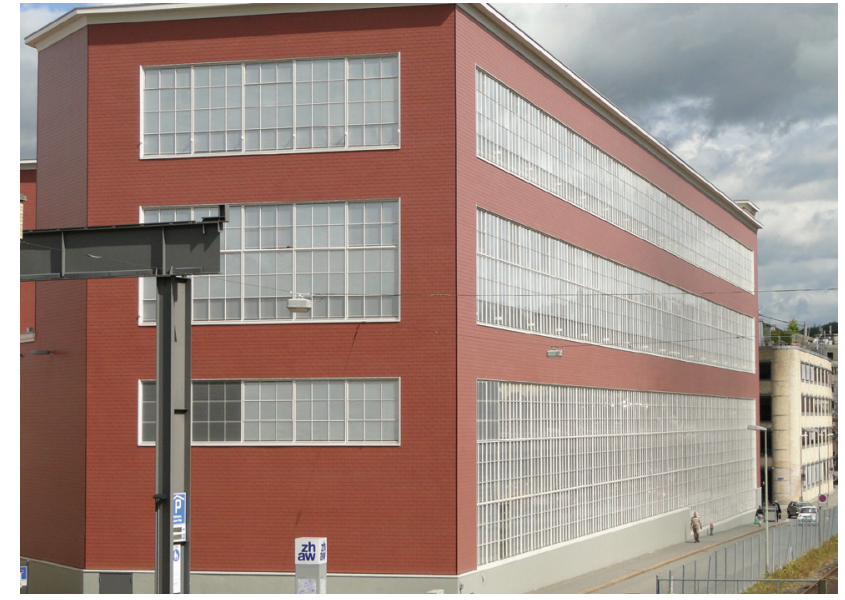

Abb. 1: Südwestansicht (Foto: W. Giella)

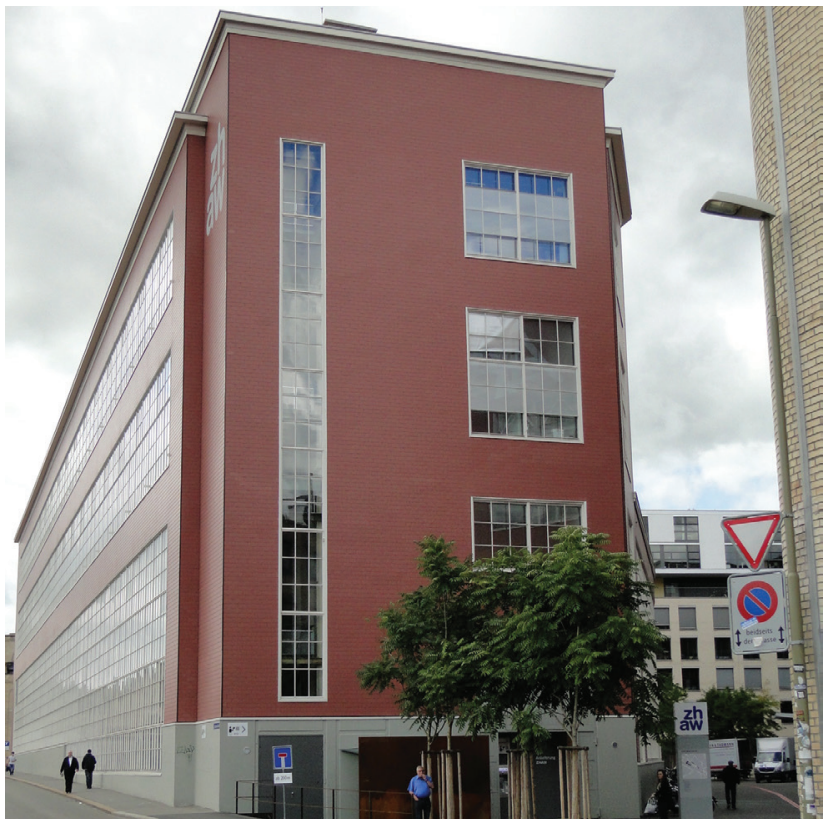

Abb. 2: Nordostansicht (Foto: W. Giella)

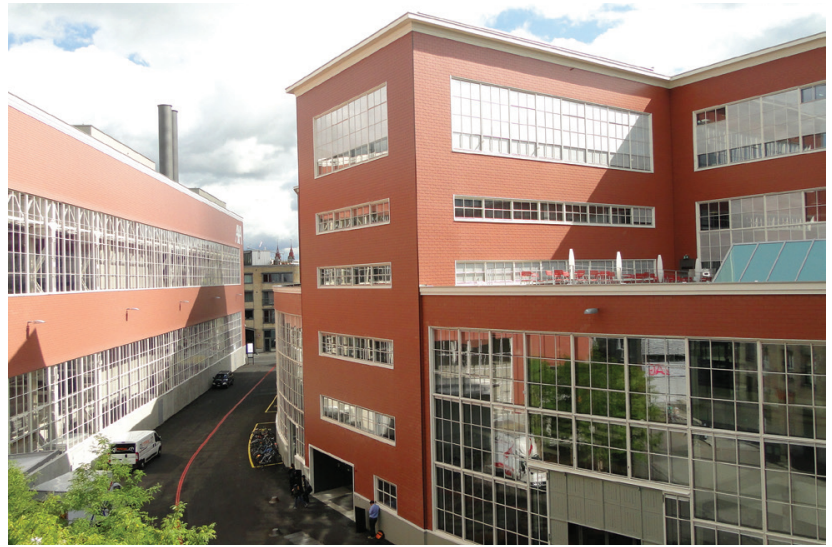

Abb. 3: Risalit (Foto: W. Giella) 
entwickelte wenig später einen Zweitaktmotor, der in der Schifffahrt die Dampfmaschine zu verdrängen begann. Es mutet schon beinahe absurd an, dass eine Firma in einer provinziell geprägten Kleinstadt eines kleinen Binnenlandes sich anschickte, den Weltmarkt mit Schiffsmotoren zu beherrschen. Noch 1930 stammte jeder dritte weltweit produzierte Schiffsmotor aus Winterthur. Der Höhepunkt wurde Mitte der 1960er Jahre erreicht, als alleine in Winterthur 14000 Menschen für Sulzer arbeiteten. Entsprechend wuchs das Sulzerareal, und auch in Oberwinterthur, einem Stadtteil Winterthurs, wurde Sulzer auf einer weiteren großen Fläche aktiv. ${ }^{8}$

Das Gebäude 87 selbst hatte einen Vorläuferbau, der wohl auf das Jahr 1900 zu datieren ist. Etwa 1929 planten die Ingenieure des firmeneigenen Baubüros der Firma Sulzer, hier einen Neubau zu errichten. Es sollte eine Rohrschlosserei und eine Ausbildungsstätte errichtet werden. Man beauftragte den Winterthurer Architekten Lebrecht Völki (1879-1937) ${ }^{9}$ mit der Planung und Gestaltung des Gebäudes. Völki beendete das Gebäude schon 1931. Im Wesentlichen bestand das Werkstattgebäude aus drei übereinanderliegenden Hallen. Völki verwendete für die Konstruktion hauptsächlich genieteten Stahl, Backstein und Eternitschindeln. Er fachte mit dem Backstein das Stahlskelett aus und konnte so großflächige Fensterfronten erstellen. Das Gebäude wurde und wird nicht durch die Fassade getragen, sondern durch eine Innenkonstruktion.

Alle drei Hallen verfügen über große Deckenkrananlagen, an denen Brückenkräne hängen. Diese Kräne sind im ganzen Gebäude als Teil des denkmalpflegerischen Inventars belassen worden. Die Erdgeschosshalle ist rund zehn, die Hallen darüber jeweils etwa fünf Meter hoch.

Heute stehen auf dem Sulzerareal immer noch etliche Industriehallen, aber nur die Halle 87 weist ihre ursprüngliche Bausubstanz auf. Dies ist der Kontext der verschärften Denkmalschutz-Auflage des „Integralen Schutzes“. Der integrale Schutz besagt im Grundsatz, dass nicht nur die Gebäudehülle, sondern auch das Innere erhalten werden soll.

Die Architekten lösten diese Herkulesaufgabe vorbildlich. Das Architekturbüro P\&B Partner Architekten $A G^{10}$ bezog in einer sehr frühen Planungsphase vor der

8 Die firmengeschichtlichen Ausführungen folgen in stark gekürzter Form den Ausstellungstexten des Historikers Miguel Garcia für die Eröffnungsausstellung der neuen Bibliothek im Februar 2015.

9 Ausführliche Informationen zum Architekten Lebrecht Völki: http://www.winterthur-glossar.ch/app/default/pub/fw.action/wine. article?ce_id=176\&ce_name=Person (07.09.2015).

10 Siehe Link: http://www.piotrowski-bovet.ch/ (10.08.2015).
Baueingabe alle Beteiligten mit ein. Der Denkmalschützer der Stadt Winterthur und die entsprechende Kommission, der kantonale Denkmalschützer, der Heimatschutz sowie die Stadtplanung wurden mehrfach zu Präsentationen eingeladen. $\mathrm{Zu}$ den vielen Auflagen auf der einen Seite stellten der Kanton und die ZHAW als Endnutzer Forderungen, welche manchmal den ästhetischen Wertevorstellungen der Architekten diametral zuwiderliefen.

Es war klar, dass die Flächenbedarfe, die der Kanton bestellt hatte, nur durch den Einbau von Zwischengeschossen im Erdgeschoss zu erfüllen waren. Eine Auflage der Denkmalpflege war aber, dass die Hallen in allen drei Stockwerken sicht- und erlebbar bleiben mussten. Also durften die Zwischengeschosse nicht durchgehend sein, sondern mussten die Sicht zur Gesamthalle gewährleisten. Nur mit Balkonen und offener Konstruktion konnte man diese Auflage nicht erfüllen, da auch feuerpolizeiliche Vorschriften (Brandabschnitte, Entrauchung und Entfluchtung) zu regeln waren. Und als Endnutzer war man stets um die akustischen Emissionen besorgt. Die Zwischengeschosse wurden schließlich wie Tische übereinander eingebaut. Bahnseitig sind sie offen, zum Verwaltungstrakt der HSB und zur Cafeteria sowie zur Gebäudegesamterschließung hin wurden riesige Glasfassaden bis zur Hallendecke hochgezogen. So wirkt das Gebäude transparent und leicht.

Die historische Gebäudehülle entspricht in keiner Weise den heutigen Dämmwerten. Die erwähnten ursprünglichen Fensterfronten übertragen jedes Außenklima in den Raum, im Sommer verstärken sie sogar die Hitze und den Lichteinfall. Auch die vernieteten Stahlträger leiten die klimatischen Außenbedingungen ungeschützt ins Gebäudeinnere und die Eternitplatten halten nur gerade den Wind fern. Im Winter versuchte man diesem Unbill mit einem Heizröhrensystem an der Innenfassade einigermaßen zu begegnen. Diese gigantisch langen Heizröhren, die als System zu sechst übereinander liegen, bewirkten aber nicht viel. An der Fassade war es heiß, zwei Meter von der Fassade entfernt herrschten beinahe Außentemperaturen. In diesem zweiten Obergeschoss bildete die Firma Sulzer ihre zukünftigen Mitarbeitenden in metallverarbeitenden Berufen aus.

Die Planung der Architekten sah vor, dass man die bestehenden großflächigen Einfachverglasungen vor Ort reparieren würde und sie wieder hell gestrichen werden würden.

Die asbesthaltigen Eternitschindeln wurden durch identische, asbestfreie Platten ersetzt. Zwischen den Platten und der Backsteinausfachung baute man eine effiziente Wärmedämmung ein. Hierfür wurden Aerogelmatten verwendet. 

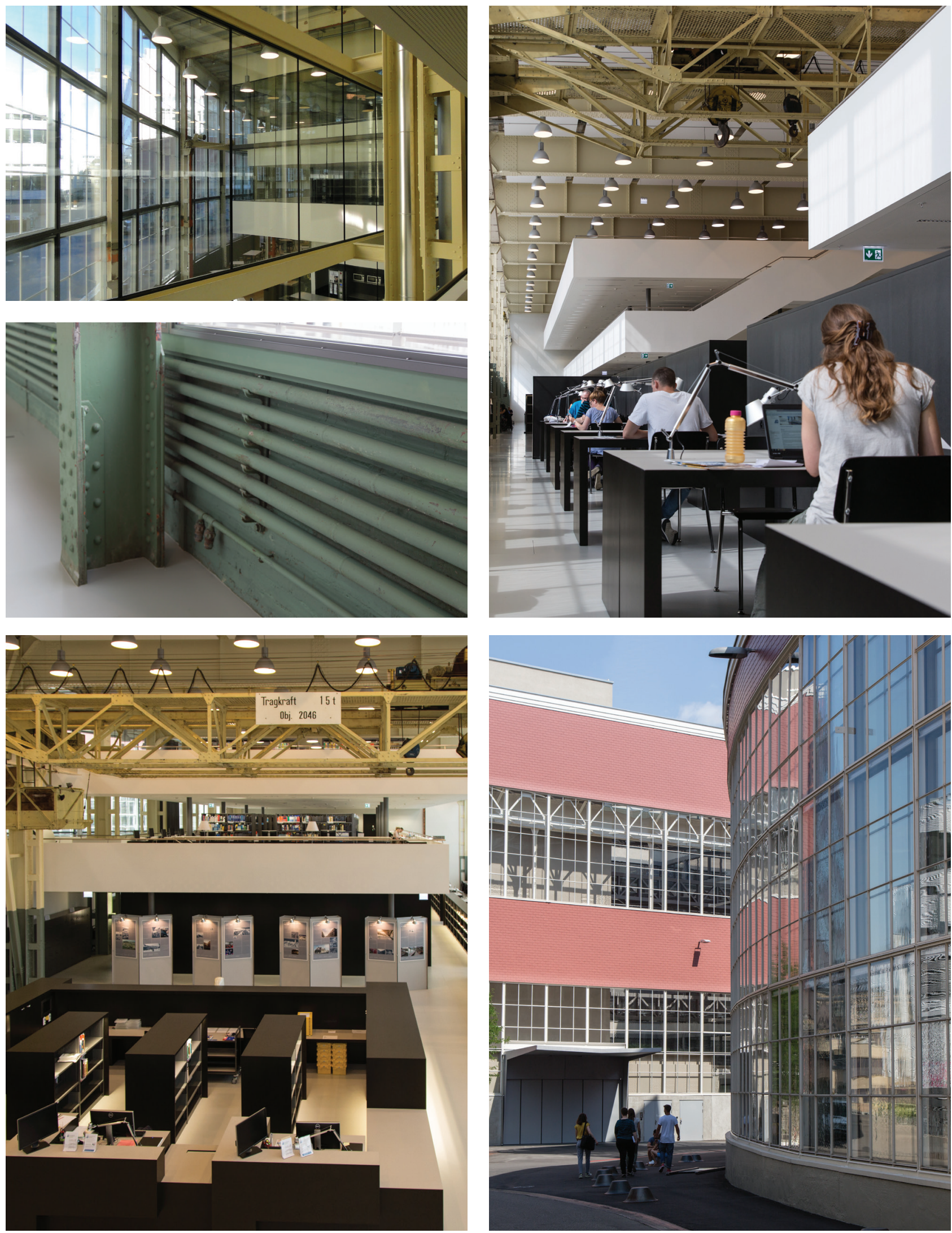

Abb. 4-8: Innenansichten mit Glasfassade, altem Heizröhrensystem, Arbeitsplätzen, Empfangstheke und Krananlage sowie Glasfassade von außen (Fotos: W. Giella, ZHAW und S. Wanke) 


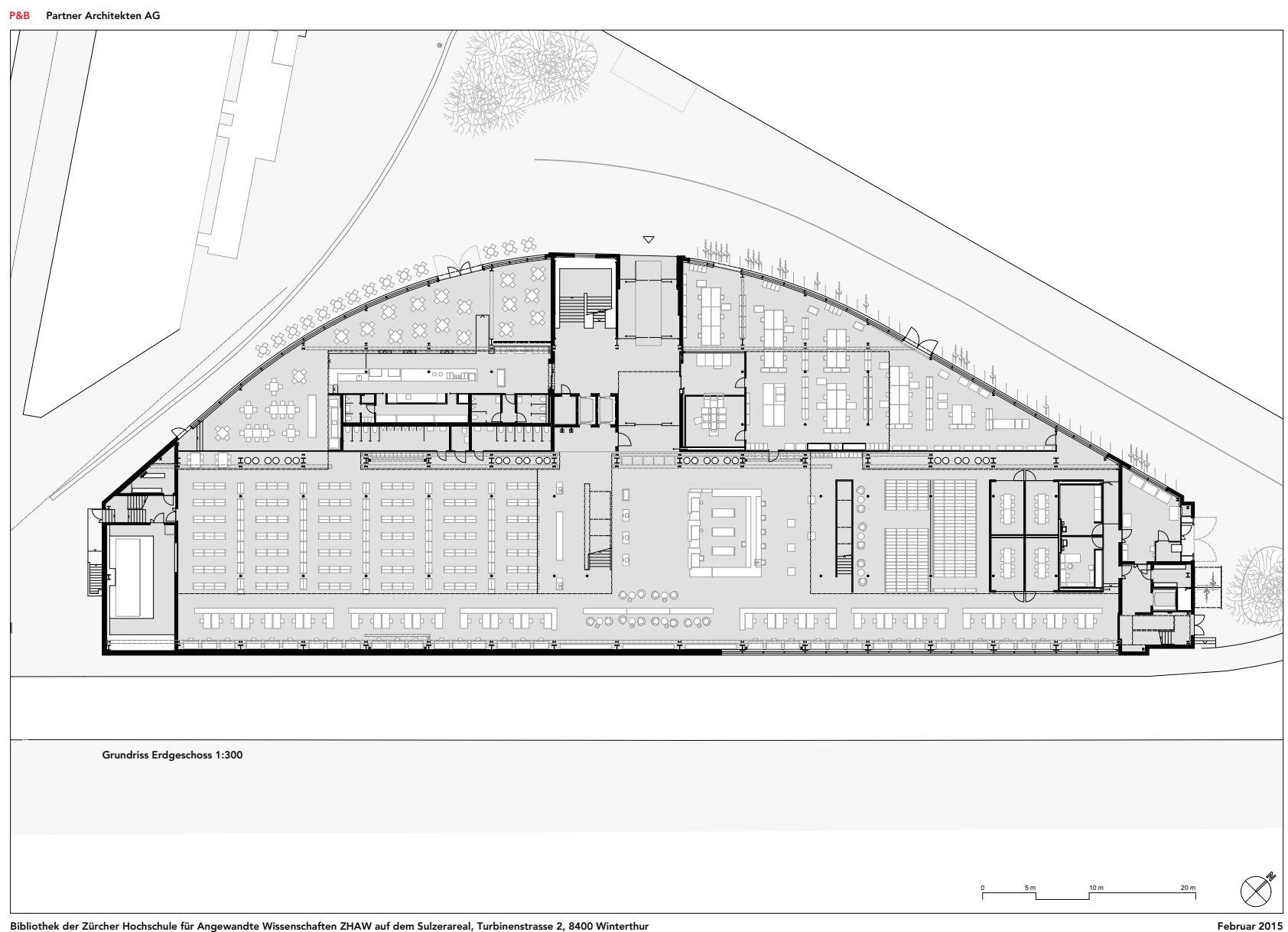

Abb. 9: Erdgeschoss (P\&B Architekten 2015)

Die enormen Glasflächen wurden im Innenbereich durch eine Dreifachverglasung gedämmt. Textile Rollos wurden als Maßnahme gegen Blendung durch Sonnenlichteinfall zwischen den Glaswänden montiert. ${ }^{11}$

\section{Die neue Bibliothek}

Der Haupteingang des Gebäudes befindet sich auf der Seite des Industrieareals und ist somit von der Bahn aus nicht sichtbar. Die Gebäudeerschließung wurde im Risalit umgesetzt. Für Hochschulangehörige mit Campuscard ist das Gebäude 365 Tage im Jahr an 24 Stunden zugänglich. Aus diesem Grund wurde der Raum mit der Selbstabholstation zwar im Innern des Gebäudes, aber noch vor dem Eingang zur Bibliothek platziert, die derzeit wochentags zwölf

11 P\&B Partner Architekten: Die Neue Bibliothek der ZHAW im Gebäude 87 auf dem Sulzerareal Stadtmitte. Erläuterungen zum Projekt. Internes Papier. Winterthur 2012. S. 3.
Stunden geöffnet ist. Im gleichen Raum können Medien auch über die Selbstrückgabe zurückgegeben werden. Gegenüber diesem bibliothekarischen Serviceraum befindet sich die rund 380 Quadratmeter große Cafeteria. Das Treppenhaus und die Lifte trennen diese beiden Bereiche.

Die Bibliothek selbst scheint auf den ersten Blick sehr klassisch zu sein. Man betritt durch Sicherungsgates die Halle, links liegt die Empfangstheke mit drei Schalterplätzen. Traditionelle Dienste wie Auskunft, Fernleihausgabe und Empfangsdienste werden hier angeboten. Für Rechercheauskünfte stehen zwei gesonderte Theken weiter oben zur Verfügung.

Rechts vom Eingang fällt die weiße Treppe ins Auge, welche die beiden Zwischengeschosse in der Bibliothek erschließt.Der Konstruktion dieser Treppe waren viele Versuche, Vorschläge und Diskussionen vorausgegangen. Schlussendlich wurde diese Konstruktion vorgezogen. Nicht nur ihre Formschönheit war der Grund. Sie schützt die Zwischengeschossbereiche vor dem „Lärm“ der Empfangstheken.

Hinter der Treppe unter dem ersten Zwischengeschoss beginnt der Freihandbereich, der sich über beide 


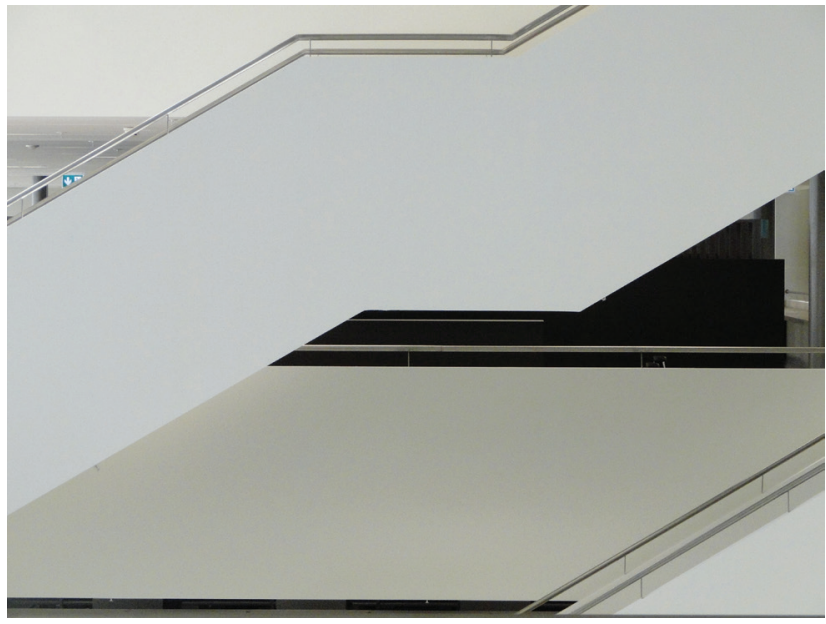

Abb. 10: Haupttreppe (Foto: W. Giella)

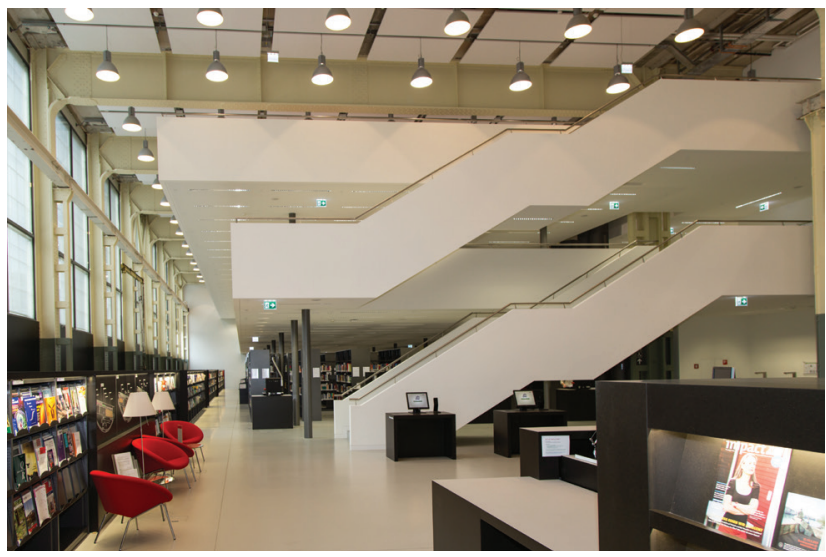

Abb. 11: Haupttreppe Gesamtansicht (Foto: S. Wanke)

Zwischengeschosse zieht und ein Soll-Medienvolumen von rund 250000 Medieneinheiten aufnehmen kann. Der aktuelle Bestand beläuft sich in Winterthur auf rund 140000 Medieneinheiten. Ein parallel zur Innenfassade laufendes Regalsystem von vier Ordnerhöhen bildet eine Trennlinie zwischen Arbeitsplätzen und Bibliothek. Diese Plätze sind bei Besuchern der Bibliothek sehr beliebt. Sie sind dank der Durchgängigkeit der Regalwand nah an den Beständen und geschützt vor Blicken und Geräuschen.

Der erwähnte modulare Planungsansatz wird im Freihandbereich der Bibliothek sichtbar, verborgener ist er in den Theken, Büros und in Teilen des zweiten Zwischengeschosses sowie in der Lernlandschaft. Bei den Regalen fällt auf, dass ihre Aufstellung eine Art von Sektoren bildet. Die Regale selbst stehen schon auf in den Boden eingelassenen Schienen. Sie können jederzeit auf Regalwagen gehoben werden und so nach Bedarf den ganzen Bereich oder einzelne Sektoren

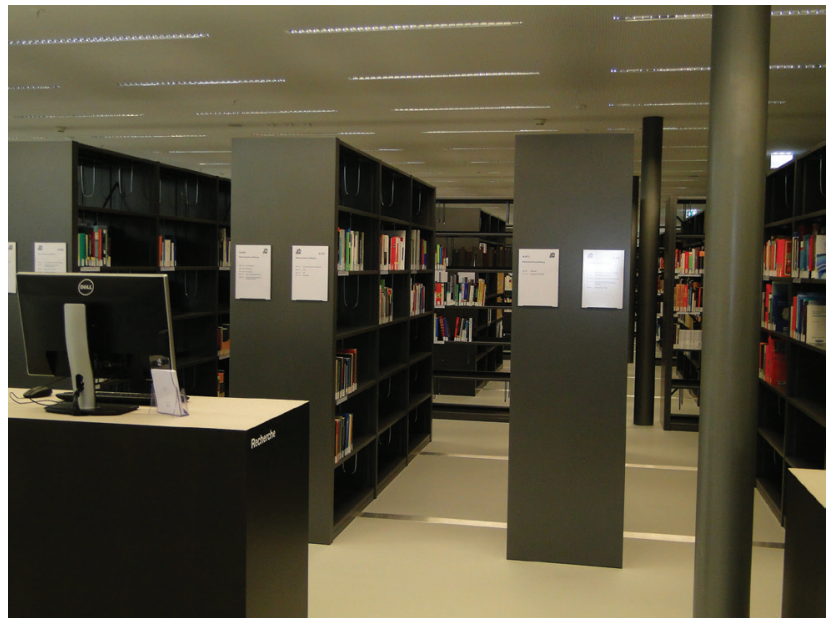

Abb. 12: Modulare Planung: In den Boden versenkte Schienen im Freihandbereich (Foto: S. Wanke)

verdichten. Trotz der Sollbestandszahl baut die ZHAW HSB vor allem auf den E-Medienbestand und Open Access-Publikationen.

Im rechten Gebäudeteil hinter der Empfangstheke durchschreitet man eine kleine Ausstellungsfläche. Eine Nebentreppe führt ins erste Zwischengeschoss hinauf. Unter diesem Zwischengeschoss stehen die Rollregale des kleinen Magazinbereichs.

Hinter diesen Rollregalen selbst befinden sich die ersten vier modern ausgestatteten Gruppenräume mit Whiteboards, Wandbildschirmen und UKV-Steckern.

Das erste Zwischengeschoss bietet am meisten Platz für den gedruckten Bestand. Dieses Stockwerk wurde nicht weiter flexibel geplant. Hier befindet sich eine der beiden Recherchetheken. $\mathrm{Zu}$ bestimmten Tageszeiten wird an diesen Theken Rechercheunterstützung hauptsächlich für Fachdatenbanken angeboten. Die meisten Bibliotheksmitarbeitenden dieser Theken sind gleichzeitig im Schulungsteam der HSB. Hinter den Recherchetheken kommt man in weitere Freihandbereiche. Arbeitsplätze in Reihe umrahmen diese und ermöglichen ein Arbeiten nahe bei den Fachbeständen.

Das zweite Zwischengeschoss hebt sich aufgrund des Bestands von den anderen beiden Geschossebenen der Bibliothek ab: Sind dort Präsenz- und Freihandbestände im geschätzten Verhältnis von zehn zu neunzig aufgestellt, so sind im zweiten Zwischengeschoss nur Präsenzbestände vorhanden, da dort der ganze Bestand des Bereichs Wirtschaftsrecht steht. Dieses Stockwerk ist ebenfalls modular geplant. Im Bedarfsfall können die Bestände massiv verdichtet und ein Flügel des Zwischengeschosses für andere Zwecke ummöbliert werden. 


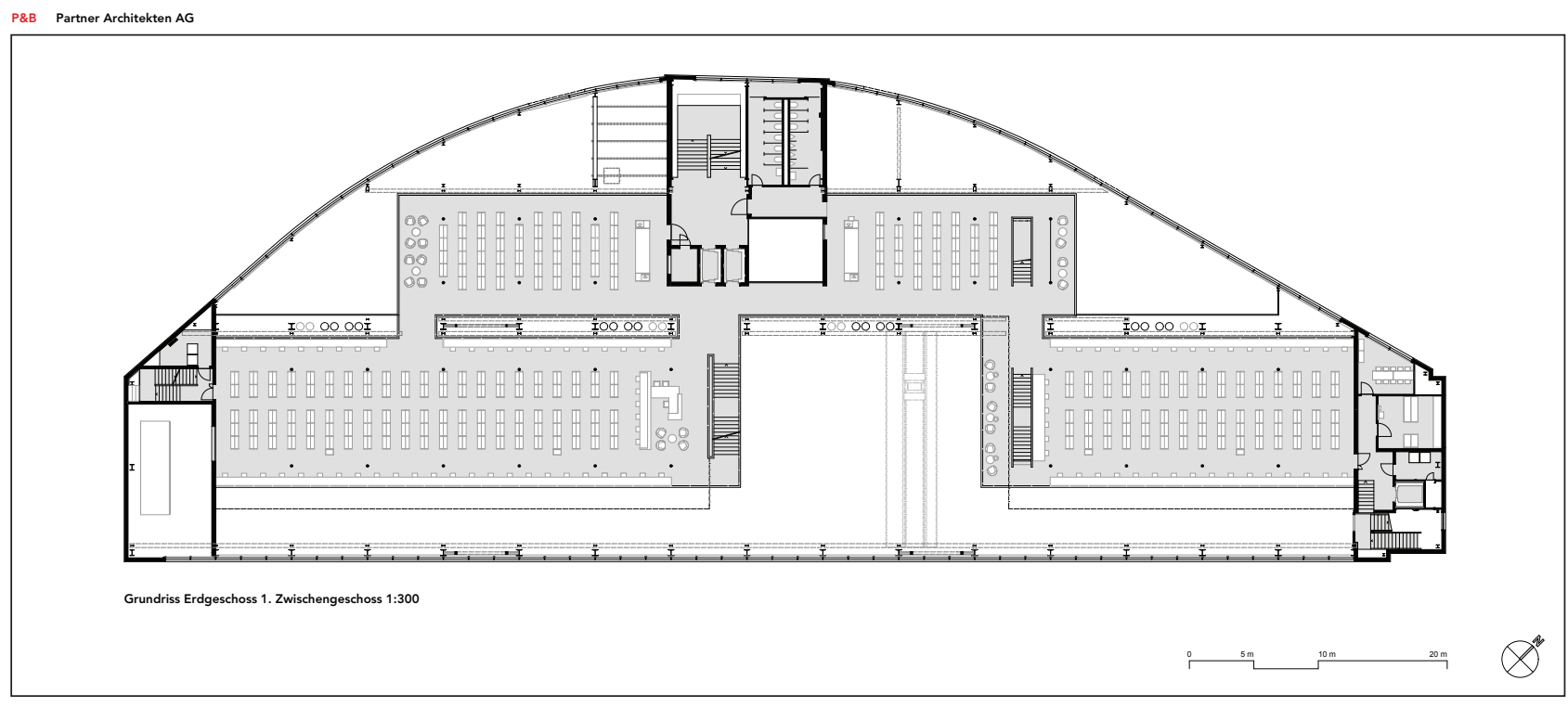

Bibliothek der Zürcher Hochschule für Angewandte Wissenschaften ZHAW auf dem Sulzerareal, Turbinenstrasse 2, 8400 Winterthur

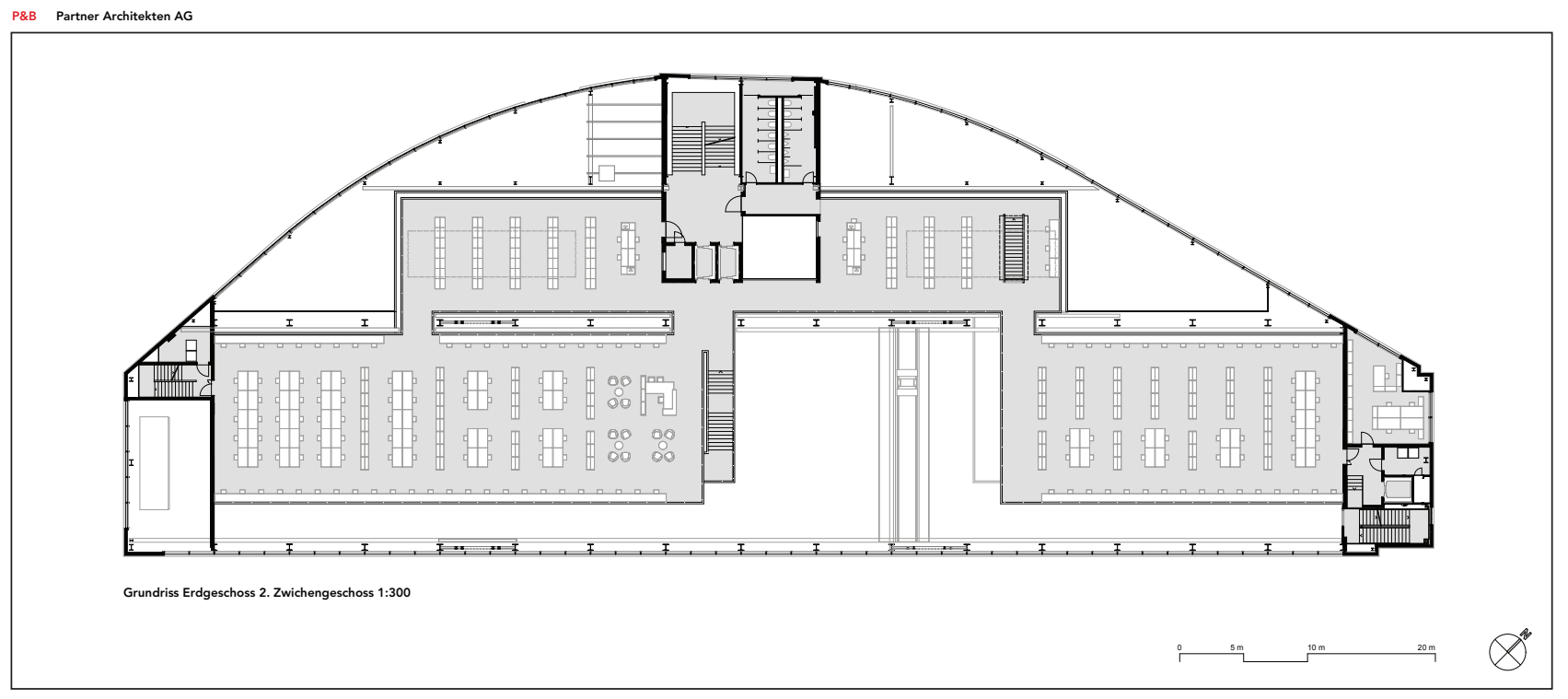

Bibliothek der Zürcher Hochschule für Angewandte Wissenschaften ZHAW auf dem Sulzerareal, Turbinenstrasse 2, 8400 Winterthur

Abb. 14: 2. Zwischengeschoss (P\&B Architekten 2015)

Alle Stockwerke sind mit Buchscannern, OPAC-Stationen und kleineren geschützten Loungebereichen ausgestattet. Kopiergeräte werden in der Bibliothek nicht angeboten, nur im zweiten Obergeschoss in der Lernlandschaft steht ein kostenpflichtiges Gerät. Die Buchscanner sind ausschließlich mit Speichersticks bedienbar, die Reproduktion ist für die Nutzenden umsonst. Durch einen hohen Pauschalbetrag an das Hochschulamt wird dem Copyright Genüge getan.

\section{Die Lernlandschaft}

Im zweiten Obergeschoss, rund achtzehn Meter über dem Boden, befindet sich die Lernlandschaft. Der Begriff der Landschaft erschien uns sehr passend: Das Mobiliar für Arbeit und Pausen, die Pflanzenwände, die Gruppenräume, die Carrels und die gewaltige Halle, die das Ganze umschließt, bilden in der Tat eine Landschaft. Diese 


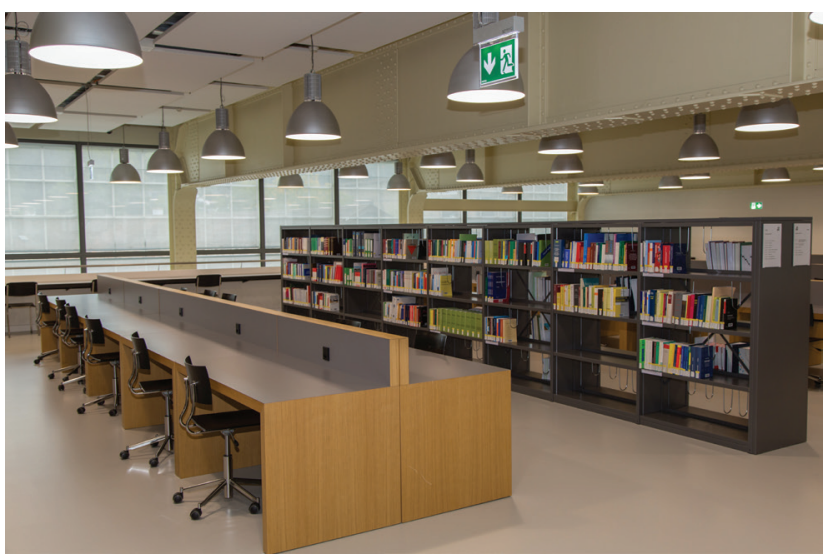

Abb. 15: Lesebereich 2. Zwischengeschoss: Deutlich ist hier die Integration bestehender Möbel aus den alten Standorten in die neue Bibliothek zu sehen (Foto: S. Wanke)

bietet damit räumliche Vielfalt für ihre Besucher, die zum Lernen und Forschen hierherkommen.

Für die große Fläche von über 1700 Quadratmetern drängte sich eine aktivitätsorientierte Konzeption auf. Jedoch wäre es vermessen zu behaupten, dass dieses Vorgehen von Anfang an so geplant war. Anfänglich sollte in Winterthur nur verhindert werden, dass steril Stühle hinter Tische gestellt und so die geforderten Arbeitsplätze im zweiten Obergeschoss abgefüllt werden. Da dieser Bereich räumlich komplett von der Bibliothek getrennt ist, verfügte man auch nicht über Erfahrungsberichte oder gar -daten anderer Institutionen mit ähnlichen räumlichen Bedingungen. ${ }^{12}$ Lernbereiche waren zu diesem Zeitpunkt in den vorab besichtigten Bibliotheken im In- und Ausland immer entweder innerhalb der Bibliothek oder zumindest in direkter Berührung mit ihr. Auch die alten und sehr unterschiedlichen Standorte in Winterthur verfügten über keine Daten, aus denen man die Wünsche und die Verhaltensweisen der künftigen Nutzenden hätte extrapolieren können.

Die Zeit drängte, und die Vorstellungen der Mitglieder des Projektteams waren sehr unterschiedlich. Die Bibliotheksleitung suchte verzweifelt nach ähnlichen Einrichtungen, aber alle sehr guten Beispiele, die es gab und gibt, ließen sich nicht wirklich vergleichen. So stellte sich bald

12 Sicherlich wäre es für uns eine große Hilfe gewesen, schon die DINIAgenda 2020 als Argumentationshilfe zur Seite zu haben. Abrufbar unter: dini.de/fileadmin/docs/dini_agenda_2020_juli2015_final.pdf.

Auch die Empfehlungen der DINI-Arbeitsgruppe „Lernräume“ kamen für unser Vorhaben ein wenig zu spät, doch bestätigten sie unser Vorgehen $\mathrm{zu}$ einer Zeit, als man noch hätte korrigieren können.Unter:http://www.uni-kassel.de/upress/online/OpenAccess/ 978-3-86219-654-8.0penAccess.pdf. die Frage, wie eigentlich berühmte Firmen ihre Arbeitsplätze gestalten. Die Konzepte von Google und Apple, der UBS, aber auch die Umsetzungen der IBM Switzerland führten bald zu neuen Fragestellungen: Was hat sich in der Großraumbüroplanung die letzten Jahre oder Jahrzehnte vollzogen und was kann man daraus lernen? Auf der Suche nach Antworten stieß man immer wieder auf die Begriffe „aktivitätsorientiert“ oder „aktivitätsbasiert“. Da die Zeit knapp war, wurde auf eine eigene akademische Ergründung dieser Begrifflichkeit verzichtet und dies den Wissenschaftlern des ZHAW Instituts für Facility Management (IFM) ${ }^{13}$ überlassen, die sich mit Workspace Research beschäftigen.

So wurde 2013 das IFM beauftragt, eine Bedarfserhebung und -analyse der Studierenden, Forschenden, Dozierenden und Bibliothekare zu erstellen. Das IFM verfügt über eine breite Erfahrung in der Workspace-Forschung, Beratungstätigkeit und Gestaltung aktivitätsorientierter Großbüroräume. Dabei ist das IFM nicht nur regional sehr bekannt, sondern arbeitet auch international.

Die zentrale Fragestellung, welche das IFM zu ermitteln hatte, wirkt recht banal: „Wie kann die physische Lern- und Arbeitsumwelt in einer Hochschulbibliothek die Lernprozesse der Studierenden effektiv unterstützen?“ Die Fragestellung wurde in dieser allgemeinen Formulierung gewählt, damit die Erkenntnisse aus dem Evaluationsprojekt nicht nur für das zweite Obergeschoss, die künftige Lernlandschaft, sondern auch zum Teil für die Bibliotheksräume im Erdgeschoss genutzt werden konnten.

Das Projekt sah eine mehrstufige Erhebung von Daten vor. Neben einer Online-Umfrage $(\mathrm{N}=760)$ unter den Studierenden der ZHAW, zwei Workshops mit weiteren Nutzergruppen und einer umfassenden Begehungsstudie wurden der aktuelle Forschungsstand sowie Umsetzungskonzepte von Großraumbüros in Banken, Versicherungen und internationalen Konzernen zu Rate gezogen.

Die Begehungsstudie erfasste die alten Standorte und erbrachte innerhalb von drei aufeinanderfolgenden Tagen 1292 Datenpunkte.

Aus den Erkenntnissen entwickelte das IFM sein Smart-Learning-Konzept, welches sich auf einen 9-ThesenKatalog stützt. „Smart Learning“ beschreibt das IFM als „aktivitätsbasiertes Konzept, welches in Lärmzonen gegliedert ist und die einzusetzenden Elemente in vier

13 Siehe Link: https://www.zhaw.ch/de/lsfm/institute-zentren/ifm/ (12.09.2015). 

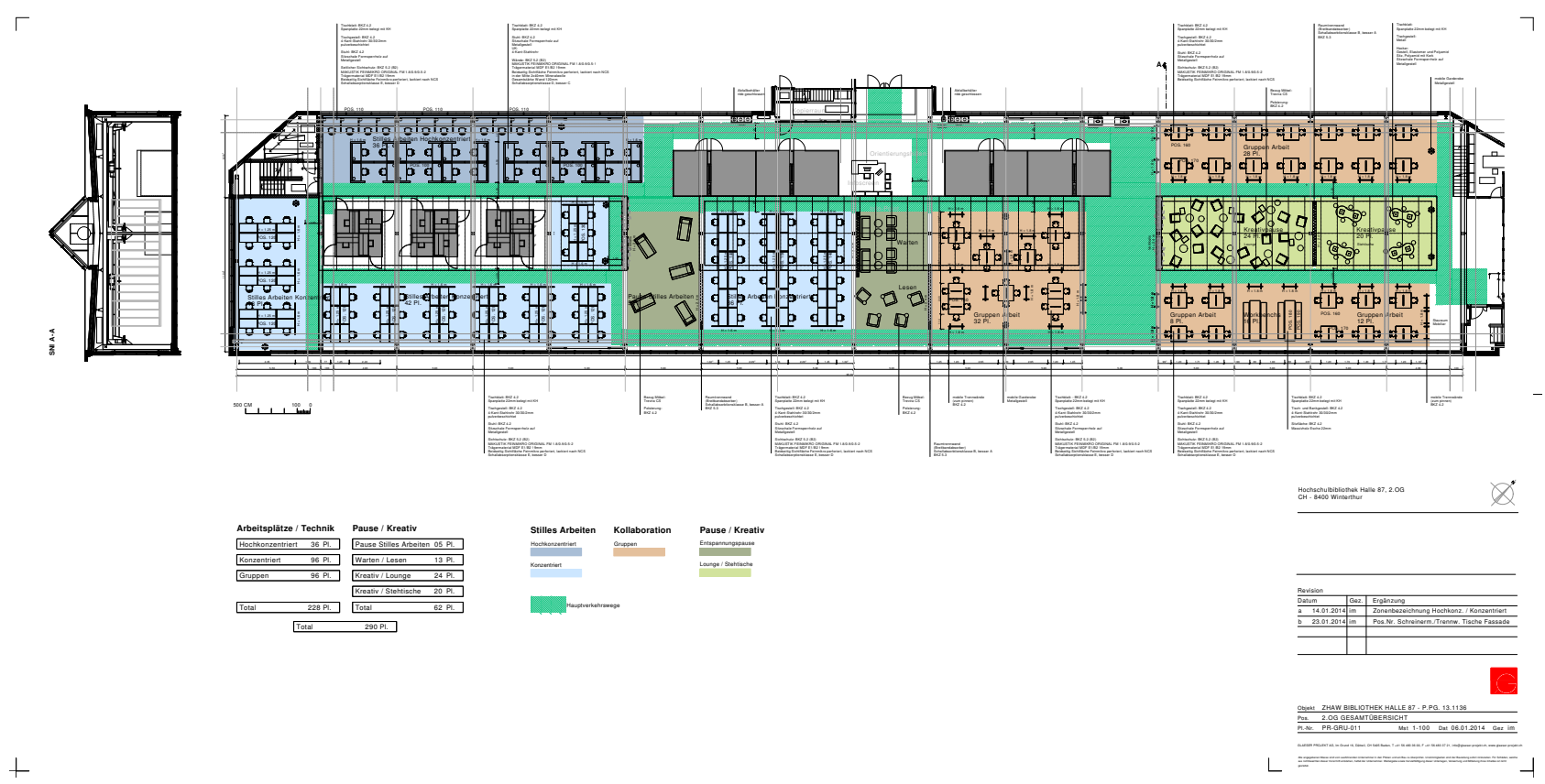

Abb. 16: Lernlandschaft zweites Obergeschoss (Françoise Chevalier und Firma Glaser 2014)

Farblegende: Dunkelblau = hochkonzentriertes Arbeiten, Hellblau = konzentriertes Arbeiten, helles Beigerot = Kollaboration, Grün = Entspannungspause, Hellgrün = Lounge und Stehtische

übergeordnete Kategorien gruppiert: stilles Arbeiten, Zusammenarbeit, Service und verschiedene Arten von Pausen." 14

Aus bibliothekarischer Sicht kann man festhalten, dass einige Thesen nicht wirklich überraschend sind, weder für die Bibliothek in Winterthur noch für andere Bibliotheken. Manche dieser vermeintlich bekannten Tatsachen haben aber durch ihre empirische Quantifizierung in ihrer Deutlichkeit erstaunt.

Ein großer Teil der Thesen ist aber zumindest neu und hat geholfen, die Ideen für die Möblierung besser zu formulieren. So beschreibt die These Nr. 2 die ungenutzten Potentiale im Bereich der Privatsphäre und Konzentration. Privatsphäre ist auch im Zeitalter von Social Media ein nicht zu vernachlässigendes Bedürfnis. Aber Privatsphäre ist durch individuelles Erleben geprägt und kann nicht für alle gleich definiert werden. Das IFM hat hier vor allem den Bereich des Sichwohlfühlens bei der konzentrierten Arbeit, beim konzentrierten Lernen in einem großen Raum abgefragt. Selbst für Gruppen besteht der Wunsch nach Privatsphäre. Aus dieser These wurde der Bedarf abgeleitet, eine Anzahl von Stellwänden zur Verfügung zu stellen.

14 Giella, Wolfgang, Jennifer Konkol, Fabienne Schanné. „Smart Learning - Förderung von effektivem Lernen durch ein aktivitätsorientiertes Raumkonzept in der Bibliothek.“ In Neue Lern- und Arbeitswelten. Orte der Kommunikation. Hrsg. von Christine Kohlert, RBS - Projekt Management GmbH. Broschüre. München: 2015. S. 43-47. Hier S. 46.

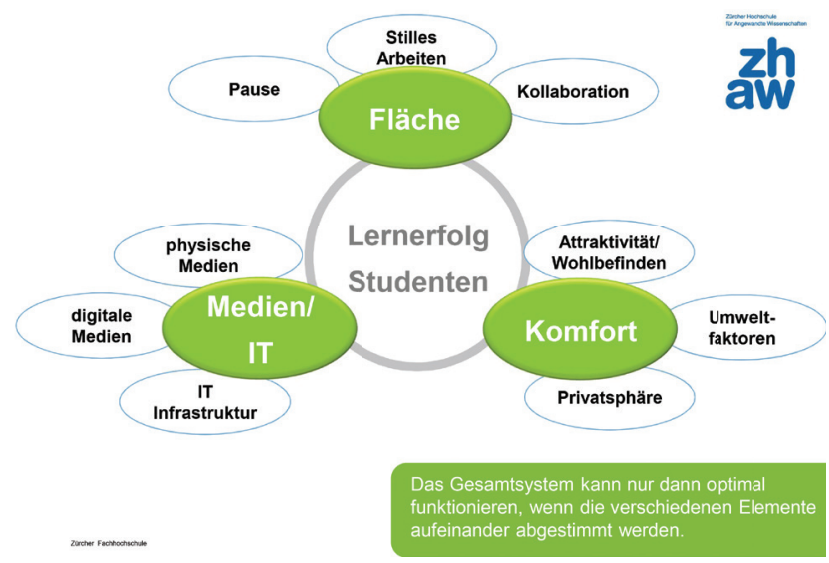

Abb. 17: Smart-Learning-Konzept des Instituts für Facility Management der ZHAW (Wädenswil 2013)

These Nr. 3 zeigt auf, dass die Generierung von Flächenvielfalt ein zentraler Erfolgsfaktor ist. Auf der individuellen Ebene zeigte sich, dass die unterschiedlichen Arbeitsstile der Studierenden sowie die breite Divergenz, wie man Erholungspausen - zusätzlich in der These 5 formuliert machen möchte, im Möblierungskonzept abgebildet werden mussten. Für Fachhochschulen und insbesondere für die ZHAW ist die These Nr. 6 interessant: Sie verrät, dass die Dozierenden die eigene HS-Bibliothek i. d. R. noch nicht für sich entdeckt haben. Abgesehen von wenigen fachlichen Ausnahmen dürfte diese These ein typisches FH-Phänomen beschreiben, welches diesen Hochschultyp 


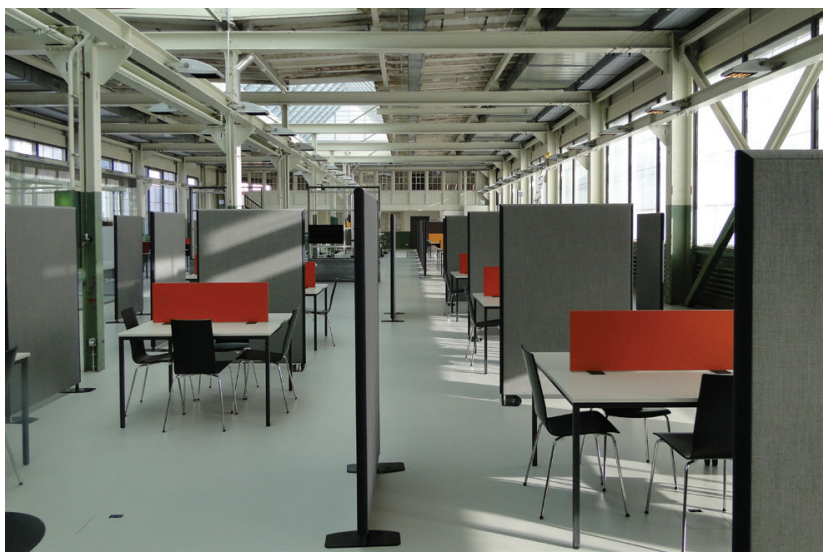

Abb. 18: Ursprüngliche Anordnung der Stellwände vor der Eröffnung (Foto: S. Wanke)

sicher von den Universitäten unterscheidet. Das liegt zumindest an der ZHAW in Winterthur bisher wohl daran, dass die HSB für einen möglichen Austausch zwischen Studierenden und Dozierenden keine geeigneten räumlichen Möglichkeiten anbot. Man muss kein Prophet sein, um zu erkennen, dass gerade diese These ein großes Potential für neue Serviceangebote von Bibliotheken beinhaltet.

Nicht unerwähnt soll These 5 bleiben, die das Bedürfnis nach Lern- und Entspannungspausen im gleichen Raumkomplex formuliert. Dies ist weder erstaunlich noch unbekannt, aber in dieser Deutlichkeit doch überraschend. Die Häufigkeit der Lernnutzung würde sich gemäß unserer Datenlage nahezu verdoppeln. Die kurze Praxiszeit hat das schon bestätigt.

Die These 7, die für alle Bibliotheksplaner und Bibliothekare bekannt und von scheinbar großer Erkenntnisbanalität ist, sagt kundenseitig erst einmal nur aus, dass IT-Infrastruktur und moderne Medien gewünscht seien. Die Thesenformulierung klingt freilich elaborierter: „IT-Infrastruktur und moderne Medien fungieren als Katalysator in Verbindung mit dem Raumkonzept.“ Dahinter steht einerseits die Begrifflichkeit „selbstgesteuertes und kooperatives Lernen“, andererseits aber auch der Wunsch nach Projekträumen. Vereinzelt wurde sogar eine Investition seitens der Bibliothek in Lernplattformen gewünscht, welche auch Telefon- und Videokonferenzen erlauben. Diese These hatte aber noch eine andere Folge in der Planung der Lernlandschaft: Der Gedanke, in dieser Lernlandschaft vielleicht eine WLAN-freie Zone zu schaffen, erwies sich als philosophische Spielerei, welche nur durch das wesentlich höhere Durchschnittsalter der Mitglieder des Projektteams erklärbar ist.

Auf der Basis dieser Thesen und des Smart-LearningKonzepts erarbeitete die Innenarchitektin ein erstes Raumkonzept für die Lernlandschaft.

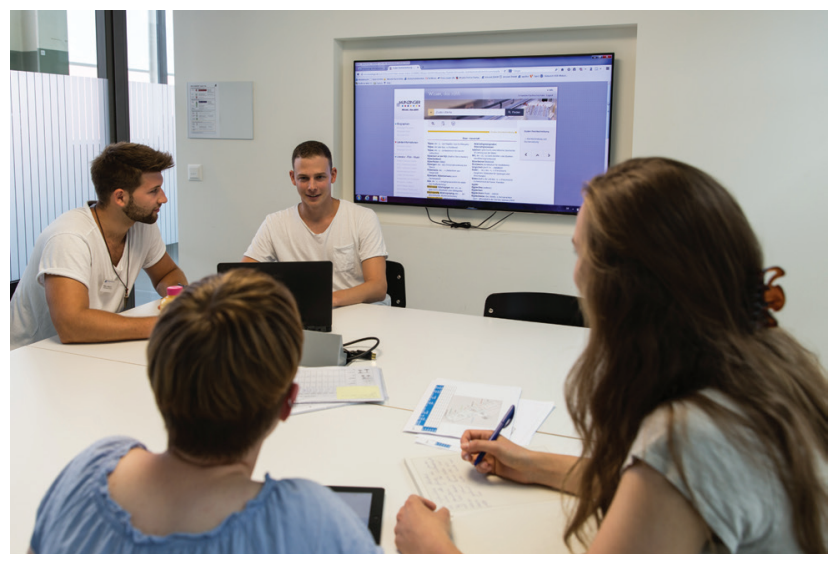

Abb. 19: Modern ausgestattete Gruppenräume. Innenansicht eines Gruppenraums in der Lernlandschaft (Foto: ZHAW)

Insgesamt ließen sich so neun Zonen um Gruppenräume und Carrels gruppieren. Die Planung der Erschließung dieser Zonen war sehr aufwendig. Es musste sichergestellt werden, dass keine wilden und störenden Pfade im Raum entstehen konnten, sondern dass sich die Erschließung des Raumes auf einem durchgängigen Hauptweg abspielt. Dieser Hauptweg bildet jeweils hallenkopfendig eine Art Kreis um die Carrels im stillen Bereich und um die Pausensitzmöbel des lauten Bereichs. Hinter den Gruppenräumen finden sich zwei Nebenerschließungspfade, ebenso an den Fensterfronten zu den Bahngleisen hin (im Plan nicht eingezeichnet).

Die Studie wurde mit einem letzten Workshop abgeschlossen, an dem der vorläufige Abschlussbericht und das erste Raumkonzept für das zweite Obergeschoss präsentiert und das Feedback aufgenommen wurden. Daraus entstand der finale Abschlussbericht durch das IFM.

Zusammenfassend kann man Folgendes festhalten: Im zweiten Obergeschoss umfasst die Lernlandschaft ein vielfältiges Raumkonzept in zwei Raumbereichen: Einen für konzentriertes oder stilles und einen für kooperatives oder lautes Arbeiten. Ein Übergangsbereich und verschiedene Maßnahmen der Möblierung sowie baulicher Art trennen diese Bereiche. Die Möbel im stillen Bereich sind schwer und unbeweglich, im lauten hingegen sind sie leicht und zu einem großen Teil mobil. Dies ermöglicht den Endnutzern, die Lernlandschaft im kooperativen Bereich jederzeit flexibel ihren Bedürfnissen gemäß zu verändern. Auch diese „baulichen Eingriffe“ in die Ordnung der Lernlandschaft durch die Endnutzer, dieses Zusammenstellen, Kombinieren, Schaffen einer individuellen Arbeitsumgebung, entspricht ebenfalls dem Bild der Landschaft. Denn hier verändert der Mensch ebenfalls die Landschaft laufend, was wir als Erfahrung nur bestätigen können. 

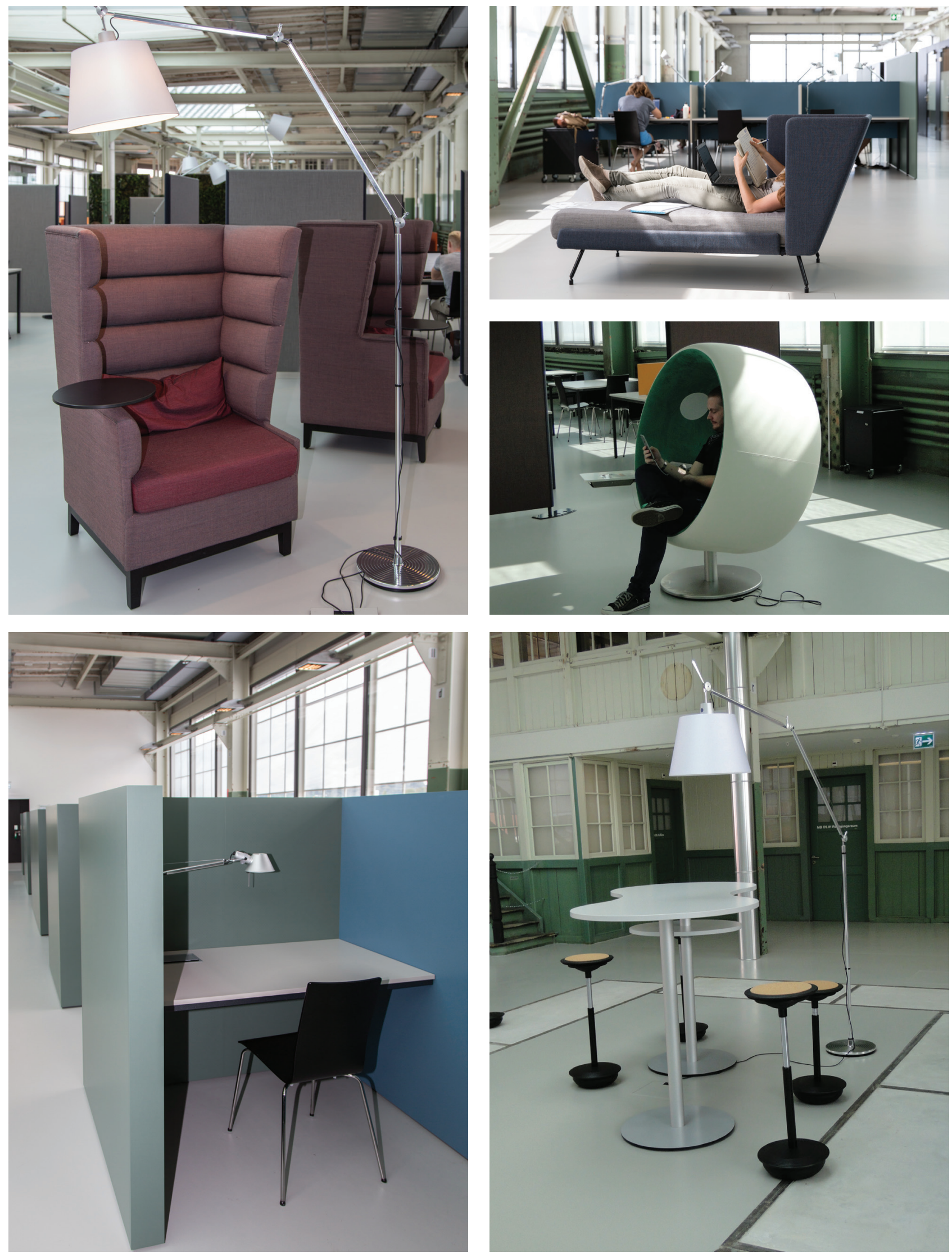

Abb. 20-24: Lernlandschaft mit verschiedenen Arbeits- und Pausenbereichen (Fotos: W. Giella, ZHAW und S. Wanke) 


\section{Die Umsetzung}

Das Raumkonzept der Innenarchitekten sowie die ermittelten Bedarfe durch das IFM ermöglichten nun, einen Kriterienkatalog zu Art und Anforderungen der einzelnen Möbel der Lernlandschaft zu erstellen. Ein Möbelplaner wurde hinzugezogen, um die verschiedene Möbeltypen $\mathrm{zu}$ besprechen. Formen und Farben der Möbel sollten nicht nur ihre Funktion, sondern auch das Raumkonzept „konzentriert versus kooperativ“ unterstützen. Für den Bereich kooperatives Arbeiten wurden drei Farbtöne im Spektrum Braun-Rot, für konzentriertes Arbeiten im Spektrum Blau-Gelb gewählt, Farben, die eine Gebäudereferenz darstellen.

Im Bereich für konzentriertes Arbeiten wurden um die Carrels Lernkabinen und verschiedenartige Leseplätze mit Sichtschutz geplant, im Bereich für kooperatives Arbeiten Polstermobiliar, leichte Tische und Holzstühle mit beweglichen Stellwänden. Diese Stellwände als Resultat der zweiten These bilden den mobilen Sichtschutz und ermöglichen den Studierenden, allein oder in Gruppen Bereiche abzugrenzen. Man kann beinahe täglich beobachten, dass die Stellwände um zusammengeschobene Tische gestellt werden und so wiederum Räume bilden. Gerade in Lern- oder Projektzeiten wird dies derart intensiv genutzt, dass die ZHAW HSB ein Aufstocken dieser Stellwände ernstlich in Erwägung ziehen muss. Vier Pflanzenwände unterstützen die Zonenunterteilung. Jede Zone verfügt über eine Pausenmöglichkeit. In der kooperativen Zone stehen Sessel mit Fußschemel und Laptophalterung. Sofas stehen in der mittleren und Chaiselongues für den kurzen Mittagsschlaf in der konzentrierten Zone.

Eine Idee aus der Bürowelt hat sich leider in der Praxis in Winterthur nicht bewährt: In der kooperativen Zone stehen vier Stehtische mit einbeinigen Stehstühlen, um Besprechungen zu ermöglichen. Aus der Forschung weiß man, dass Besprechungen auf diese Art und Weise wesentlich effizienter sind als in einer klassischen Sitzungsbestuhlung. Allerdings scheinen das die angehenden Jungforscher nicht auf sich zu beziehen, denn die Plätze sind fast immer unbesetzt.

Insgesamt entstand in der Lernlandschaft ein Angebot mit über 320 Arbeitsplätzen, das nach zwei ersten Auslastungserhebungen zu Prüfungszeiten und einer großen Feedbackanalyse nur wenige Wünsche offen lässt. Gäste aus den USA und Australien meinten gar, dass sie ein solches Angebot auch aus ihren Ländern nicht kennen würden.

\section{Reaktionen und erstes Fazit}

Bei Führungen konnte festgestellt werden, dass diese Bibliothek beeindruckt. Selbst in der eher zurückhaltenden Schweiz, wo man sich gegenseitig nicht mit Lob zu überhäufen pflegt, erreichen die Bibliothek sehr positive Rückmeldungen. Eine der schönsten ist sicher die von Herbert Staub, dem Verbandspräsidenten des BIS Bibliothek Information Schweiz. In einer E-Mail vom 14. März 2015 schreibt er:

„Wenn ich Bibliothekar wäre, oder gar Bibliotheksdirektor, wäre ich jetzt wahrscheinlich neidisch auf ... die tolle Hochschulbibliothek. Weil ich weder das eine noch das andere bin, kann ich mich einfach freuen - und als Präsident von BIS ... bin ich stolz auf die neuen Dimensionen, die in Winterthur zu sehen sind. Ich gratuliere ... zum erreichten Ergebnis; ich ... bin mir sicher, dass Bibliotheksreisen fortan nicht mehr nur in die skandinavischen Länder gehen, sondern auch nach Winterthur. Oder wieder nach Winterthur - die Stadtbibliothek gehörte ja ebenfalls zur Avantgarde der Schweizer Bibliothekslandschaft." 15

Noch sind nicht alle Mängel behoben. Die Bibliothek und die Lernlandschaft werden aber sehr gut genutzt und es lässt sich schon eine positive Zwischenbilanz ziehen, die durch eine Bachelorarbeit ${ }^{16}$ des IFM bestätigt wird. Mittels einer ausführlichen Begehungsstudie sowie zusätzlichen Leitfadeninterviews und einer Feedback-Wand wurde die allgemeine Zufriedenheit in der Prüfungszeit Ende Mai, Anfang Juni 2015 ermittelt. Die nonverbale Zufriedenheit zeigt sich an der durchschnittlichen Gesamtauslastung von 90 Prozent. In der Prüfungszeit ist jedoch das vielfältige Angebot der Lernlandschaft nicht gefragt, 95 Prozent der Nutzenden verrichteten konzentriertes Arbeiten (Vorbereitungen für Prüfungen, Abschlussarbeiten verfassen), nur 5 Prozent arbeiteten kooperativ. Das war aber zu erwarten. Selbst sonntags war die Auslastung bei 75 Prozent, und nachts erfassten wir durch Handzählung eine Auslastung rund 35 Prozent (Zählung an drei Nächten um 23.30 Uhr). Auch ist die Fremdnutzung durch Studierende der Universität Zürich oder der ETH recht hoch, wie durch eigene Befragungen erhoben wurde.

15 E-Mail vom 14.03.2015.

16 Mischler, Janine: Flächennutzungsstudie und Bewertung der neuen ZHAW Lernlandschaft in Winterthur. Bachelorarbeit am Institut für Facility Management der ZHAW in Wädenswil. Eingereicht am 11. August 2015. 


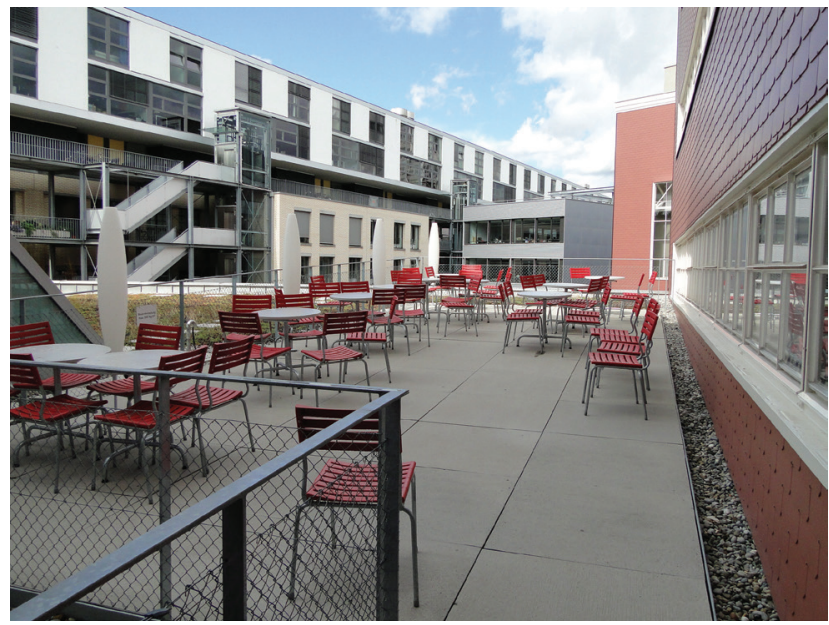

Abb. 25: Pausen im Sommer: Eine der beiden Dachterrassen (Foto: W. Giella)

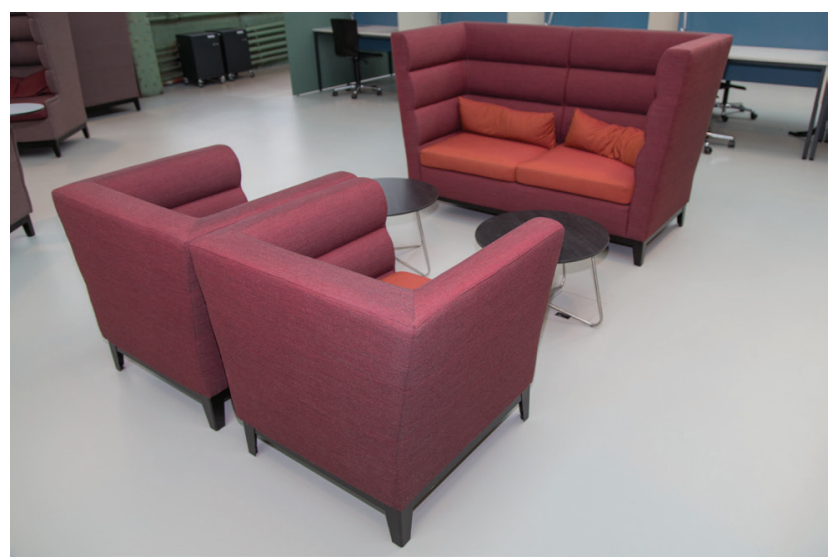

Abb. 26: Sofasitzgruppe der Lernlandschaft (Foto: S. Wanke)

Die Zufriedenheit (N=284) lag gemäß der Feedback-Wand bei 75 Prozent (davon 62 Prozent sehr zufrieden), 23 Prozent gaben an, unzufrieden zu sein (10 Prozent sehr unzufrieden). Allerdings bezogen sich diese Unzufriedenen nicht auf die Angebote der Lernlandschaft, sondern zu einem sehr großen Teil auf die damals noch nicht eingespielte Lüftung.

Aus einer internen ersten Erhebung Anfang Mai ist zudem bekannt, dass sich ein Teil der Nutzenden phasenweise bis zu 14 Stunden täglich in der Lernlandschaft aufhält.

Abschließend kann man sagen, dass man - wenn man die Räumlichkeiten hierfür zur Verfügung hat aktivitätsbasierte Konzepte umsetzen sollte; sie sind ein sicherer Weg zum Erfolg und - das wurde bisher noch nicht erwähnt - durch diese Planung können mehr Arbeitsplätze auf der gleichen Fläche produziert

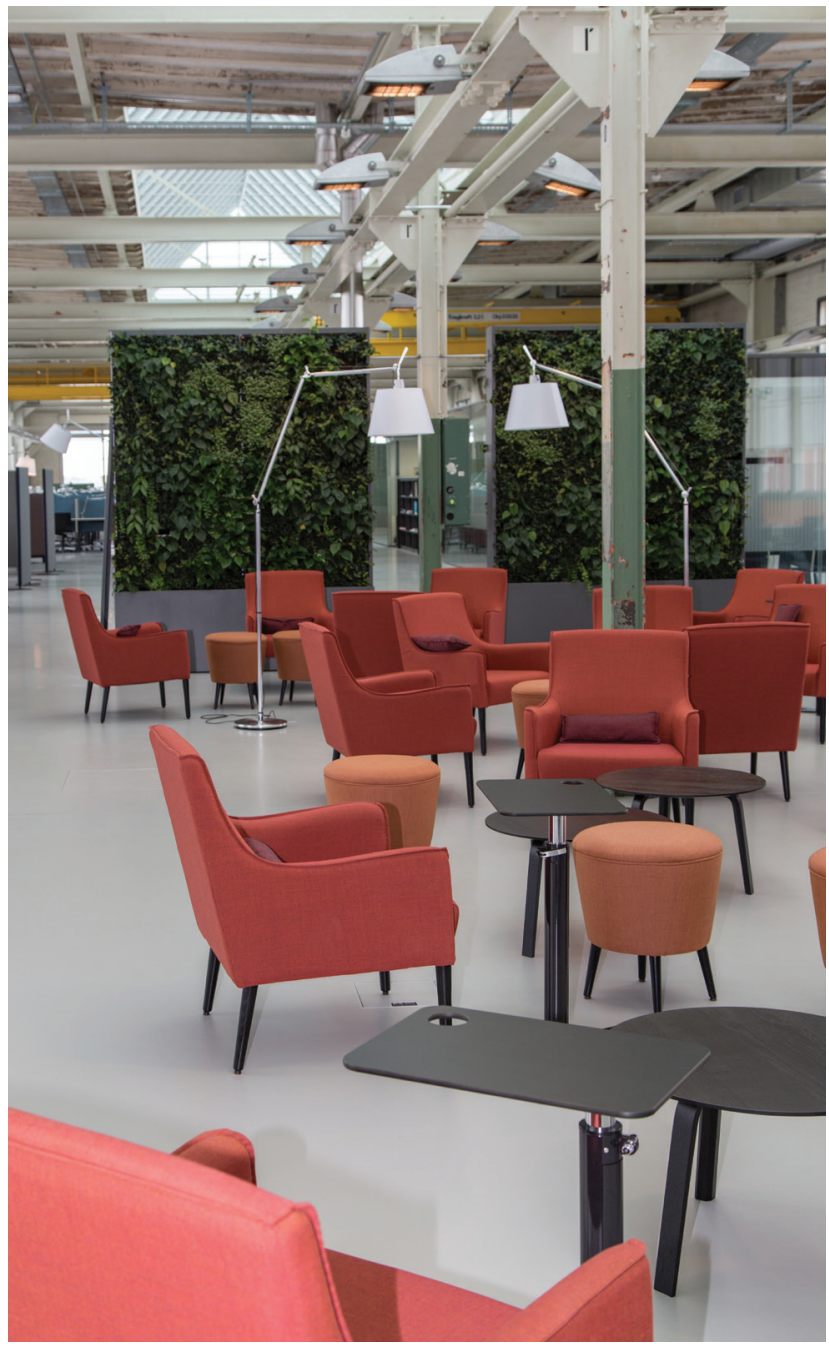

Abb. 27: Pausenbereich vor Pflanzenwänden (Foto: S. Wanke)

werden als durch konventionelle Planung. Dennoch ist der Raumeindruck freier, weniger beengt. Der Blick in die moderne Büroplanung für die Konzeption einer Hochschulbibliothek scheint abwegig zu sein. In Wahrheit ist er bereichernd. Das bestätigt das erfolgreich abgeschlossene Projekt in Winterthur.

\section{Autoreninformationen}

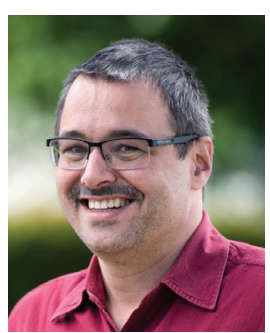

Dr. Wolfgang Giella

ZHAW Finanzen \& Services

Turbinenstrasse 2

$\mathrm{CH}-8400$ Winterthur

+41(0) 589346587

wolfgang.giella@zhaw.ch

orcid.org/0000-0001-5512-8464 